\title{
Nursing Students' Medication Errors and Adherence to Medication Best-Practice
}

\author{
Ibrahim Salami \\ School of Nursing, The University of Jordan, Amman, Jordan \\ Email: i.salami@ju.edu.jo
}

How to cite this paper: Salami, I. (2018) Nursing Students' Medication Errors and Adherence to Medication Best-Practice. Open Journal of Nursing, 8, 281-291. https://doi.org/10.4236/ojn.2018.85024

Received: April 19, 2018

Accepted: May 8, 2018

Published: May 11, 2018

Copyright $\odot 2018$ by author and Scientific Research Publishing Inc. This work is licensed under the Creative Commons Attribution International License (CC BY 4.0).

http://creativecommons.org/licenses/by/4.0/

\section{c) (i) Open Access}

\begin{abstract}
Background: Nursing students are at risk for committing medication administration errors (MAEs), which significantly alter the delivery of safe and effective healthcare. Purpose: To identify the medications most frequently involved in medication errors as reported by Jordanian nursing students, as well as to identify the level of nursing students' adherence to best-practice when administering high-risk medications. Methods: A cross-sectional design was used among a convenience sample of 74 nurses. Results: A total of 74 of 110 (67.3\%) questionnaires were returned. The most frequent medications subjected to MAEs were Dopamine, Dobutamine, and Insulin continuous intravenous infusion (17.2\%, 16.0\% and $13.5 \%$ respectively). Regarding the adherence to best-practice, nursing students adhered the most to the following best-practices: checking the patient armband prior to medication administration with a mean of $3.81( \pm 0.6)$ and bringing the MAR sheet with them when preparing a medication with a mean of $3.46( \pm 1.1)$. Conclusion: Developing an effective medication training programs in all undergraduate nursing programs in Jordanis is vital to ensure patient safety. Nursing educators in clinical and academic settings need to reinforce the importance of adherence to medication management best-practice in all courses. Close and effective supervision of students needs to be maintained throughout nursing students' clinical training, especially during medication preparation and administration.
\end{abstract}

\section{Keywords}

Medication Administration Error, Nursing Students, Medication

Best-Practice, Jordan

\section{Introduction}

Medication management is a high-risk, high-frequency nursing task in everyday practice [1]. It involves the processes of prescription, transcription, dispensing, 
preparation and administration of medication [2]. Providing opportunities for students to become competent in medication management is a core goal of all undergraduate nursing educational programs [3]. Medication competence requires a solid knowledge base in pharmacology, mathematical calculation [1], and psychomotor skills in medication preparation [1] [4]. Deficiencies in nursing students' medication competence, as well as limited clinical experience significantly increase the risk of medication errors [5].

Medications are part of the patient treatment plan, and appropriate management is critical to ensure patient safety [6]. Any medications could cause harm if given in the wrong concentration, at the wrong frequency, or via the wrong route [7]. If high-alert medications are given in error, they could cause harm more frequently, therefore increasing patient suffering and maximizing the cost of care [6] [7]. Medication error has been defined as any preventable event that may lead to inappropriate use of medication or cause temporary or permanent patient harm [8].

Many international studies have been conducted to measure the incidences, causes and contributing factors to Medication Administration Errors (MAEs) among nursing students. These studies have concluded that the incidence of MAEs among nursing students was surprisingly high and frequently occurred [4] [9] [10]. In their cross-sectional survey Cebeci et al. [9] found that $38.8 \%$ of nursing students had made at least one MAE during their clinical training. Additionally, they found that the most common cause of MAEs among nursing students was performance deficit, while the most common contributing factor was workload. Other studies found that medication errors committed by students were caused by practicing without full supervision from the bedside registered nurse [11].

In their fourth year of education, undergraduate nursing students at the University of Jordan become more independent in their clinical training and practice without constant supervision from their clinical preceptors. In their last semester, students work 52 shifts in their preferred clinical area shadowing the bedside registered nurses. The transition from directly supervised clinical training to independent self-directed bedside training exposes patients to medication errors committed by nursing students.

Therefore, the aim of this study was to identify the medications most frequently involved in MAEs as reported by Jordanian nursing students. In addition, a secondary objective of this study was to identify the level of nursing students' adherence to best-practice when administering high-risk medications.

\section{Method}

This cross-sectional study was conducted from December 2016 to December 2017 after approval by The University of Jordan-School of Nursing Institutional Review Board. Based on a $\mathrm{G} \times$ Power analysis [12] version 3.0.10 (with $\alpha$ 0.05, power 0.80 , and medium effect size) the sample required was 60 participants. A 
convenient sample of full time nursing students at their last university semester without pervious bedside nursing experience was recruited for this study. In addition, students should be already completed 40 out of the 52 shifts required for their clinical training course. During a regular student meeting, the research assistant introduced himself to the students, explained the purpose of the study to all students, and then answered questions and concerns. Upon approval, the questionnaire package was provided to each potential participant. The package included an introductory letter, informed consent, the questionnaire, and a return envelope. Participants were asked to complete the questionnaire independently and were given 40 minutes on average to complete the questionnaire. The researchers' reassured participants that access to the questionnaire would be tightly controlled by the researchers, and no names of students or clinical areas would be revealed in any form in order to maintain their confidentiality.

The first section of the used questionnaire collected demographic data from nursing students, which included the following information: student age, gender, hospital type, clinical training unit, and type of high school diploma earned. The second section asked nurses to list the most frequent medications they have difficulties with during medication management (preparing, calculating, mixing or administrating). The third section asked for nurses to use a 5-point Likert scale format from 1 = never to 5 = always to rank their adherence to best-practice when administering high-risk medications, factors that promoted compliance with the protocol, factors that could increase the risk of medication error, the extent of their familiarity with the protocol, and confidence with the protocol [13]. A detailed description of the questionnaire used in this research, as well as the psychometric properties including validity and reliability were presented in the research conducted by Salami et al. [13].

Data were analyzed using the Statistical Package for the Social Sciences (SPSS) version 21.0. (SPSS Inc., Chicago, IL, USA). Data were presented using mean, percentage, standard deviation, and proportion for categorical variables.

\section{Results}

\section{1) Sociodemographic characteristics}

A total of 110 questionnaires were disseminated and 74 of those were returned (67.3\% response rate). Table 1 describes the demographic data of the sample. $79.7 \%$ of participants were female, their average age was $22.4( \pm 0.66)$ and ranged from 22 - 24 years old. These nurses did their clinical training in three kinds of hospitals: governmental, teaching, and private hospitals. The highest rate of participation came from students trained in Intensive Care unit (ICU) departments.

2) The most frequent medication involved in MAEs as reported by Jordanian nursing students:

Nursing students reported that the medications most frequently subjected to MAEs during medication management (preparing, calculating, mixing, or 
Table 1. Sociodemographic characteristics $(n=74)$.

\begin{tabular}{|c|c|c|}
\hline Sociodemographic variable & Number (\%) & Mean (SD) \\
\hline Age (in years) & & $\begin{array}{c}22.4( \pm 0.66) \\
\text { Range }(22-24)\end{array}$ \\
\hline \multicolumn{3}{|l|}{ Gender } \\
\hline Female & $59(79.7 \%)$ & \\
\hline Male & $15(20.3 \%)$ & \\
\hline \multicolumn{3}{|l|}{ Clinical training Hospital type: } \\
\hline Governmental & $34(45.9 \%)$ & \\
\hline Private & $19(25.7 \%)$ & \\
\hline Teaching & $21(28.4 \%)$ & \\
\hline \multicolumn{3}{|l|}{ Clinical training unit: } \\
\hline Medical floor & $11(14.9 \%)$ & \\
\hline Surgical floor & $16(21.6 \%)$ & \\
\hline Intensive Care Unit (ICU) & $25(33.8 \%)$ & \\
\hline Emergency Department (ER) & $8(10.8 \%)$ & \\
\hline Maternity & $4(5.4 \%)$ & \\
\hline Pediatric floor & $2(2.7 \%)$ & \\
\hline \multirow[t]{2}{*}{ Others } & $6(8.1 \%)$ & \\
\hline & $2(2.7 \%)$ & \\
\hline \multicolumn{3}{|l|}{ Type of high school } \\
\hline Scientific & $67(90.5 \%)$ & \\
\hline Health & $7(9.5 \%)$ & \\
\hline
\end{tabular}

administering) were Dopamine, Dobutamine, I.V. Insulin, Potassium chloride, Heparin I.V. infusion, S.Q. insulin, Morphine, Amiodarone, Gentamicin and Chemotheray, in respective order of frequency. More details can be found in (Table 2).

3) Adherence to best-practice when administering high-risk medications:

Nursing students adhered the most to the following aspects of best-practice: checking the patient armband prior to medication administration with a mean of $3.81( \pm 0.6)$ and bringing the MAR sheet with them when preparing a medication (mean $3.46( \pm 1.1)$ ). One of the primary areas of interest in this study was examining the level to which nursing students adhered to best-practice during the medication management process. However, students also reported participating in practices that increased the risk for medication errors such as: preparing and carrying medications for more than one patient at the same time with a mean of $3.66( \pm 0.8)$, and administering medication that another nurse has prepared with a mean of $3.22( \pm 1.4)$ (Table 3$)$.

\section{Discussion}

This study is the first study conducted on nursing students' MAEs in Jordan. 
Table 2. Medications most frequently involved in MAEs as reported by Jordanian nursing students.

\begin{tabular}{ccc}
\hline Medications most frequently involved in & Number & Percentage (\%) \\
MAEs ( $\mathbf{n}=\mathbf{7 4}$ ) & 28 & $17.2 \%$ \\
Dopamine (Intropin) & 26 & $16.0 \%$ \\
Dobutamine (Dobutrex) & 22 & $13.5 \%$ \\
Insulin Intravenous (IV) continuous infusion & 20 & $12.3 \%$ \\
Potassium chloride (KCL) & 18 & $11.0 \%$ \\
Heparin I.V. continuous infusion & 16 & $9.8 \%$ \\
Insulin Subcutaneous (S.Q) injection & 12 & $7.4 \%$ \\
Morphine & 9 & $5.5 \%$ \\
Amiodarone (Cordarone) & 8 & $4.9 \%$ \\
Gentamicin (Gentamicin) & 4 & $2.4 \%$ \\
\hline
\end{tabular}

*The number of medications most frequently involved in MAEs exceeds the sample size number since each nurse had the chance to report up to 10 medications.

Table 3. Adherence to best-practice when administering high-risk medications.

\begin{tabular}{|c|c|}
\hline Best-practice behavior: & Mean (SD) \\
\hline Do you check the patient's armband prior to administer medication? & $3.81( \pm 0.6)$ \\
\hline $\begin{array}{l}\text { *Do you prepare and carry medications for more than } 1 \text { patient with you at a } \\
\text { time? }\end{array}$ & $3.66( \pm 0.8)$ \\
\hline Do you label the medication cup with the patient's name \& room number? & $2.94( \pm 1.2)$ \\
\hline $\begin{array}{l}\text { Do you bring the Medication Administration Record (MAR) sheet with you } \\
\text { when you prepare a medication? }\end{array}$ & $3.46( \pm 1.1)$ \\
\hline $\begin{array}{l}\text { Do you label syringes and infusions with the medication, name, patient name, } \\
\text { and room number? }\end{array}$ & $3.15( \pm 1.2)$ \\
\hline${ }^{*}$ Do you administer medication that another nurse has prepared? & $3.22( \pm 1.4)$ \\
\hline $\begin{array}{l}\text { Do you have unusual doses independently-double checked by another nurse? } \\
\text { (e.g: heparin) }\end{array}$ & $3.06( \pm 1.1)$ \\
\hline Do you have insulin doses independently-checked by another nurse? & $3.16( \pm 1.0)$ \\
\hline Do you check the patient allergies before administering medication? & $3.38( \pm 1.4)$ \\
\hline $\begin{array}{l}\text { Do you have a direct supervision from the bedside Registered Nurse }(\mathrm{RN}) \text { while } \\
\text { preparing and administering medications? }\end{array}$ & $2.84( \pm 0.6)$ \\
\hline
\end{tabular}

${ }^{*}$ Negatively stated behaviors, reverse coded in the analysis.

Among the 74 students who participated in this study, 48 (64.9\%) declared to have committed at least one MAE during their undergraduate education period. The relatively moderate rate of reported MAEs among nursing students in this study could be even higher. Underreporting may be caused by students' unwillingness to admit error due to a fear of receiving a low grade [9].

In Jordan there are no systems or policies for reporting MAEs, and hospitals do not usually disclose the actual rate of MAEs for fear of affecting their reputa- 
tions. The Jordanian healthcare system is composed of 65 private hospitals, 15 military hospitals, 32 governmental hospitals, and two university-affiliated teaching hospitals [14]. At the same time there are 15 universities with nursing bachelor's degree programs and 4 community colleges that grant associate's degrees in nursing. This large number of hospitals should provide the essential clinical training for nursing students in all nursing practices, especially in medication management. Sadly, this is not the case. The majority of private hospitals charge tuition from each student to allow them to practice in their units. Governmental hospitals suffer from overcrowding of patients, visitors, and all kinds of students. Additionally, shortages in resources and clinical nursing educators are a common complaint in governmental hospitals. As a result, each university must send their nursing students in high numbers to a limited number of hospitals with a clinical instructor from the university without any guidance from the hospital staff and without having a preceptor or a clinical educator from the hospital side easing and coordinating the clinical practice for students. All these factors together result in minimizing the learning opportunities available in nursing students' clinical practice.

1) Adherence to best-practice when administering high-risk medications:

\section{a) Checking patient ID}

There are variations in the literature regarding the compliance with checking patient ID among bedside registered nurses. Gill et al. [15] reported a low rate of adherence to checking patient ID prior to medication administration. Additionally, they found that checking patient ID prior to medication administration was highly influenced by many factors such as the nurse's perception of potential harm, type of medication being administered, the nurse's familiarity with the medication or patient, and the age of the patient. Similarly, Dickinson et al. [16] reported that checking patient ID was not always consistent among nurses, even among those working in the same units. This finding was consistent with the result of this study, in which there was a significant number of nursing students who reported noncompliance with this basic safety practice in medication management.

b) Bringing the medication sheet (MAR) when preparing the medication

The second highest reported practice prior to administering medications was bringing the medication sheet (MAR) when preparing the medication. Even though this practice is an essential part of medication administration protocol, there are still a significant portion of nursing students who are noncompliant with this best-practice protocol. The significance of bringing the MAR sheet to the medication room or patient room prior to medication administration was not investigated in any previous studies.

\section{c) Double checking}

There are very few studies that have investigated the issue of double checking prior to medication administration, and no studies investigated that issue as it relates to nursing students. In this study, there were two items asking nursing students about the practice of double checking: "Do you have unusual doses in- 
dependently-double checked by another nurse?" and "Do you have insulin doses independently-checked by another nurse?" The level of adherence to these two practices was on the average level of mean $3.06 \pm 1$ and $3.16 \pm 1.0$ consecutively.

Again, there are controversies in the literature on the significance of double checking. Armitage [17] reported that double checking may contribute to MAEs because of the checkers' time limitations, and the sense of reduced responsibility in relying on the second nurse. On the other hand, Cohen [18] reported that people checking the work of others will find about $95 \%$ of all mistakes.

d) The availability of direct bedside nursing supervision

The majority of nursing students in this study reported the absence of a bedside nurse's direct supervision during medication management. This could be explained because nursing instructors from the schools of nursing have a large number of students in the same clinical area or in different scattered areas. Therefore, students may be administering medications on their own, or they may have been given the authority to do so by the bedside registered nurse.

This result was not surprising, Reid-Searl et al. [5] reported in their qualitative study on the importance of direct nursing students' supervision that in all cases of medication errors, nursing students reported the absence of required direct supervision. In the same way, Susan [11] reported that the best defense against nursing students' MAEs is the availability of high levels of supervision, guidance, and support that students received from bedside RNs.

2) The most frequent medication involved in MAEs as reported by Jordanian nursing students.

All research studies that have investigated the levels of medication knowledge and MAEs have been conducted on bedside nurses with a wide variety of experiences. No studies were found that have assessed the medications most frequently subjected to MAEs among nursing students. Therefore, the discussion of our results will be compared to results from studies conducted on bedside RNs.

In an investigation of intravenous medication (I.V.) medication error events among critical care nurses, Bagheri-Nesami et al. [19] identified heparin to be the most frequent medication subjected to MAEs (11.5\%), followed by insulin $(3.8 \%)$ in the fourth place, and potassium chloride in the seventh place (3.4\%). Nurses in this study had a working experience of $9.3 \pm 5.87$ years. In our study, nursing students did not have any previous experience in nursing. They identified dopamine, dobutamine, and insulin I.V. infusion to be the top three medications involved in their MAEs (Table 2). Another survey conducted by the United States Pharmacopoeia (USP) [20] found that insulin was again the number 1 medication (4\%) to be misused or mishandled among all healthcare professionals. Potassium chloride (2.2\%) was in the third place followed by heparin $(1.7 \%)$ in the fifth place.

\section{Limitations}

Based on the researcher literature search this is the first study to investigate the medication error among Jordanian nursing students. Keeping in consideration 
that this study was conducted on students studying at the University of Jordan School of nursing only, other students at other nursing programs in different Jordanian universities were not included in this study. In addition, fear of receiving a low grades may have been contributing to non-reporting the MAEs in some students. Subsequently, the issue of MAEs among Jordanian nursing students may be greater than reported in this study. Finally, the findings must be considered in the context of self-reported recalled medication error during clinical training period, which extends over three years. Therefore, it may not be appropriate to generalize the findings to different educational settings.

\section{Conclusions}

The findings of this study suggested that Jordanian nursing students are at high risk of committing MAEs. In addition, it found that noncompliance with medication administration best-practice was widely prevalent among nursing students.

This study provides nursing educators in both academic and clinical settings with a better understanding of the weaknesses that nursing students have in medication management. This study also provides invaluable knowledge about the level of nursing students' adherence to medication management best-practice. Moreover, the results of this study should guide nursing educators to reinforce students' clinical training on pharmacology, policy, and procedures of medication management.

Having a well-organized clinical training program on medication management as well as reinforcing a high level of supervision in all Jordanian undergraduate nursing programs is paramount to ensure high quality nursing preparation that maintains patients' safety.

\section{Acknowledgements}

This research was supported and sponsored by The University of Jordan.

\section{References}

[1] Sulosaari, V., Huupponen, R., Hupli, M., et al. (2015) Factors Associated with Nursing Students' Medication Competence at the Beginning and End of Their Education. BMC Medical Education, 15, 223. https://doi.org/10.1186/s12909-015-0513-0

[2] Oshikoya, K.A., Oreagba, I.A., Ogunleye, O.O., et al. (2013) Medication Administration Errors among Paediatric Nurses in Lagos Public Hospitals: An Opinion Survey. International Journal of Risk \& Safety in Medicine, 25, 67-78.

[3] Asensi-Vicente, J., Jimenez-Ruiz, I. and Vizcaya-Moreno, M.F. (2017) Medication Errors Involving Nursing Students: A Systematic Review. Nurse Educator, 1-5. https://doi.org/10.1097/NNE.0000000000000481

[4] Koohestani, H.R. and Baghcheghi, N. (2009) Barriers to the Reporting of Medication Administration Errors among Nursing Students. Australian Journal of Advanced Nursing, 27, 66-74. 
[5] Reid-Searl, K., Moxham, L. and Happell, B. (2010) Enhancing Patient Safety: The Importance of Direct Supervision for Avoiding Medication Errors and Near Misses by Undergraduate Nursing Students. International Journal of Nursing Practice, 16, 225-232. https://doi.org/10.1111/j.1440-172X.2010.01820.x

[6] International JC, Organizations JCoAoH (2014) Joint Commission International Accreditation Standards for Hospitals. Joint Commission Resources, Oakbrook Terrace.

[7] Hsaio, G.Y., Chen, I.J. and Yu, S., et al. (2010) Nurses' Knowledge of High-Alert Medications: Instrument Development and Validation. Journal of Advanced Nursing, 66, 177-190. https://doi.org/10.1111/j.1365-2648.2009.05164.x

[8] Kim, K.S., Kwon, S.H., Kim, J.A., et al. (2011) Nurses' Perceptions of Medication Errors and Their Contributing Factors in South Korea. Journal Nursing Management, 19, 346-353. https://doi.org/10.1111/j.1365-2834.2011.01249.x

[9] Cebeci, F., Karazeybek, E., Sucu, G., et al. (2015) Nursing Students' Medication Error and Their Opinion on the Reasons of Error: A Cross-Sectional Survey. The Journal of the Pakistan Medical Association, 65, 457-462.

[10] Gregory, D., Guse, L., Davidson, D.D., et al. (2009) What Clinical Learning Contracts Reveal about Nursing Education and Patient Safety. The Canadian Nurse, 11, 20-25.

[11] Susan, L.P. (2017) To Err Is Human: Understanding Medication Errors amongst Nursing Students in Singapore. 6th Annual Global Healthcare Conference, New York, 15-16 February 2017, 69-78.

[12] Faul, F., Erdfelder, E., Lang, A.G., et al. (2007) GPower 3: A Flexible Statistical Power Analysis Program for the Social, Behavioral, and Biomedical Sciences. Behavior Research Methods, 39, 175-191. https://doi.org/10.3758/BF03193146

[13] Salami, I., Subih, M., Al-Jbarat, M., et al. (2018) Medication Administration Errors: The Perceptions of Jordanian Nurses. Journal of Nursing Care Quality.

[14] Ministry of Health (2018) Health Sector Guide. http://moh.gov.jo/Pages/viewpage.aspx?pageID=195

[15] Gill, F., Corkish, V., Robertson, J., et al. (2012) An Exploration of Pediatric Nurses' Compliance with a Medication Checking and Administration Protocol. Journal of Specialists in Pediatric Nursing, 17, 136-146. https://doi.org/10.1111/j.1744-6155.2012.00331.x

[16] Dickinson, A., McCall, E., Twomey, B., et al. (2010) Pediatric Nurses' Understanding of the Process and Procedure of Double-Checking Medication. Journal of Clinical Nursing, 19, 728-735. https://doi.org/10.1111/j.1365-2702.2009.03130.x

[17] Armitage, G. (2009) The Risks of Double Checking. Nursing Management, 16, 30-35. https://doi.org/10.7748/nm2009.05.16.2.30.c7012

[18] Cohen, H. (2007) Reduce the Risks of High-Alert Drugs. Nursing, 37, 49-55. https://doi.org/10.1097/01.NURSE.0000287726.78164.af

[19] Bagheri-Nesami, M., Esmaeili, R. and Tajari, M. (2015) Intravenous Medication Administration Errors and Their Causes in Cardiac Critical Care Units in Iran. Materia Socio-Medica, 27, 442-446.

[20] United States Pharmacopeia (2018) Web Site. http://www.usp.org 


\section{Appendix I: Questionnaire}

\section{Section I. Medication Administration Practice Questionnaire.}

\section{Demographics:}

1. The name of the hospital you are currently practicing at:

2. The Unit you are currently practicing at:

3. Date of entry to the nursing program (dd/mm/yyyy):

4.

Expected year of graduation

5.

Type of high school

6.

Age (in years)

7.

Gender

Male

Female

8.

University cumulative GPA

\begin{tabular}{|c|c|}
\hline A & $\begin{array}{l}\text { Number of Medication error you did during your career history, according to the } \\
\text { following categories: (Medication rights-related ME) }\end{array}$ \\
\hline 1. & Giving a medication to the wrong patient \\
\hline 2. & Giving a medication in a wrong concentration \\
\hline 3. & Giving a medication in the wrong route \\
\hline 4. & Giving a medication in the wrong amount \\
\hline 5. & Giving a medication on the wrong time \\
\hline 6. & Giving the wrong medication \\
\hline
\end{tabular}

B Number of Medication error you did during your career history, according to the following categories: (Route-related ME)

$\begin{array}{cc}\text { 1. } & \text { Oral } \\ \text { 2. } & \text { S.C. } \\ \text { 3. } & \text { I.M. } \\ \text { 4. } & \text { I.V. direct push } \\ \text { 5. } & \text { Continuous Infusion } \\ \text { 6. } & \text { Other routes } \\ & \text { (if applicable :) }\end{array}$

C According to your experience, what are the medications you frequently have a hard time working with (Preparing, calculating. Mixing, or administrating)? Please list:

1.

2.

5.
7.

10. 


\section{Section II. Medication Administration practice.}

\begin{tabular}{ll}
\hline Think of the medications that you have & About \\
administered during your carrier & Always Frequently half the Rarely Never \\
history, how frequent: & time
\end{tabular}

1. Do you check the patient's armband prior to administer medication?

2. Do you prepare and carry medications for more than 1 patient with you at a time?

3. Do you label the medication cup with the patient's name \& room number?

Do you bring the medication

4. administration record (MAR) sheet with you when you prepare a medication?

Do you label syringes and infusions with

5. the medication, name, patient name, and room number?

6.

Do you administer medication that another nurse has prepared?

Do you have unusual doses

7. independently-double checked by another nurse? (e.g: heparin)

8. Do you have insulin doses independently-checked by another nurse?

9. Do you check the patient allergies before administering medication?

Do you have direct supervision from the

10. bedside Registered Nurse (RN) while preparing and administering medications? 